\title{
Shot Noise in Gyroklystrons
}

\author{
Thomas M. Antonsen, Jr. and Wallace M. Manheimer, Fellow, IEEE
}

\begin{abstract}
Expressions for the shot noise generated in the input cavity of a gyroklystron are calculated. Results are given for noise amplitude, noise temperature, and phase noise. For a beam of uncorrelated electrons the noise temperature scales as the beam energy. An estimate of the role of collective effects on the noise properties is given. These might either decrease or increase the noise level. It is expected that the shot noise in an actual device will depend in a sensitive way on the profile of magnetic field in the compression region preceding the input cavity.
\end{abstract}

Index Terms-Electrostatic cyclotron instability, gyroklystron, noise.

\section{INTRODUCTION}

A HIGH power $94 \mathrm{GHz}$ gyroklystron is now being developed at the Naval Research Laboratory (NRL), ultimately for application in a millimeter wave radar system [1]. In modern radar systems, it is important to keep the transmitter noise as low as possible. For the gyroklystron, which is a multiple cavity amplifier, this means minimizing the noise in the input cavity, since this is the noise which is amplified as it progresses through the various cavities. There are a number of sources of noise which can effect electron beam devices [2]. These may be classified as intrinsic or extrinsic depending on their source. Intrinsic noise results from processes directly associated with the formation and transport of the electron beam. Examples include shot noise which is associated with the discreteness of the electronic charge, flicker noise which is associated with imperfections in the emission process, and ion oscillation noise which is associated with the unstable interaction between the electron beam and ions trapped in the beam region. Extrinsic source noise results from fluctuations in the external power supplies attached to the device. The effect of these has been examined for gyroklystrons in [3]. This paper will be concerned exclusively with shot noise.

As a result of the discreteness of the electronic charge, the beam current entering the first cavity is not steady in time, but has a high frequency fluctuation spectrum which excites a signal in the cavity. The level of the signal depends on the spectrum and amplitude of the current fluctuations as well as the mean parameters of the electron beam and those of the cavity. As we shall see, these latter parameters serve to define an effective resistance which along with the fluctuating part of

Manuscript received December 17, 1997; revised March 3, 1998. This work was supported by the Office of Naval Research.

T. M. Antonsen, Jr. is with the Naval Research Laboratory, Washington, DC 20375-5320 USA. He is also with the Science Applications International Corporation, McLean, VA 22102, and the Departments of Electrical Engineering and Physics, University of Maryland, College Park, MD 20742 USA.

W. M. Manheimer is with the Naval Research Laboratory, Washington, DC 20375-5320 USA.

Publisher Item Identifier S 0093-3813(98)04197-6. the beam current determines the noise power in the cavity. The effective resistance, given in (15), depends on the geometry of the cavity, the coupling between the cavity mode and the beam, and the velocity pitch ratio of the spiraling electron beam. A typical value is about $100 Q \Omega$.

The level of current fluctuations depends on the degree of correlation of the electrons as they enter the cavity. The simplest expression for "bare" shot noise assumes that all of the particles in the electron beam are uncorrelated with one another. In this sense, the "bare" shot noise is a very fundamental quantity to calculate. It is certainly a useful quantity to know as one starts to do noise measurements on the input cavity. Under the assumption of uncorrelated electrons we shall find that the noise power spectrum in the cavity corresponds to a noise temperature of the order of the beam energy. Various physical processes can increase or decrease the shot noise level by inducing correlations among the electrons. It could be decreased by electrostatic shielding of long wavelength fluctuations (dependent on beam temperature and geometry) or by some active beam conditioning. It could be increased by electrostatic instabilities in the beam tunnel. The possibility of these effects will also be discussed in this paper. Calculation of the "bare" shot noise is thus the point of departure for more refined theories which take into account dielectric shielding and beam instabilities. We hope this work will prove useful in interpreting initial noise experiments and in stimulating future theoretical studies.

\section{Calculation of Shot Noise in the INPUT CAVITY OF A GYROKLYSTRON}

The input of a gyroklystron is coupled to the microwave source through a combination of waveguides and coupling cavities. This excites a signal in the bunching cavity of some strength which is amplified from cavity to cavity. However, there is also noise (current fluctuations) on the electron beam. These fluctuations also excite a signal in the bunching cavity which will compete with the desired signal. One cause of the noise is that the electron beam is not a continuous stream of charge, but rather, a random stream of electrons which enter the input cavity at some rate. This is the shot noise. The purpose here is to calculate the shot noise under a variety of assumptions.

We now sketch out a derivation of the basic equations. The cavity fields are governed by Maxwell's equations which we write in the Fourier domain

$$
\begin{aligned}
& \nabla \times \widehat{\boldsymbol{B}}=\frac{4 \pi}{c} \hat{\boldsymbol{J}}-i \frac{\omega}{c} \hat{\boldsymbol{E}} \\
& \nabla \times \widehat{\boldsymbol{E}}=i \frac{\omega}{c} \hat{\boldsymbol{B}}
\end{aligned}
$$


where the hatted variables are the Fourier transforms with frequency $\omega$ of the real time dependent fields. We now assume that the fields in the cavity are dominantly those of a single mode of the cavity. The mode eigenfunction satisfies (1a) and (1b) with $\hat{\boldsymbol{J}}=0$, and satisfies the boundary condition that tangential components of electric field and normal components of magnetic field vanish on the cavity walls. This eigenfunction is denoted by $\boldsymbol{E}_{\boldsymbol{n}}$, and $\boldsymbol{B}_{\boldsymbol{n}}$, where the eigenfrequency is $\omega_{n}$, and we choose the normalization of the electric and magnetic fields to be given by

$$
\int d^{3} x\left|\boldsymbol{E}_{n}\right|^{2}=\int d^{3} x\left|\boldsymbol{B}_{\boldsymbol{n}}\right|^{2}=V
$$

where $V$ is the volume of the cavity. Thus, $\boldsymbol{E}_{\boldsymbol{n}}$ and $\boldsymbol{B}_{\boldsymbol{n}}$ are dimensionless. Further, we have used the fact that the electric and magnetic fields of a resonant cavity mode have the same stored energy.

The actual, time dependent field in the cavity is given approximately by

$$
\boldsymbol{B}(\boldsymbol{x}, t)=\int_{0}^{\infty} \frac{d \omega}{2 \pi} A(\omega) \boldsymbol{B}_{\boldsymbol{n}}(x) e^{-i \omega t}+\text { c.c. }
$$

and

$$
\boldsymbol{E}(\boldsymbol{x}, t)=\int_{0}^{\infty} \frac{d \omega}{2 \pi} A(\omega) \boldsymbol{E}_{\boldsymbol{n}}(x) e^{-i \omega t}+\text { c.c. }
$$

where $A(\omega)$ is a complex scalar amplitude to be determined. Here we have introduced the single sided Fourier integral, which when added to its complex conjugate is equivalent to the normal Fourier integral for the real functions $\boldsymbol{E}(\boldsymbol{x}, t)$ and $\boldsymbol{B}(\boldsymbol{x}, t)$. This step is taken so that in subsequent operations only positive values of frequency need to be considered.

Inserting $\widehat{E}=A \boldsymbol{E}_{\boldsymbol{n}}$ and $\widehat{B}=A \boldsymbol{B}_{\boldsymbol{n}}$ into (1a) and (1b) and using a few standard vector manipulations gives [4], [5]

$$
A(\omega)=-\frac{2 \pi i \int d^{3} x \boldsymbol{E}_{n}^{*} \cdot \hat{\boldsymbol{J}}}{V\left(\omega-\omega_{n}+i \omega_{n} / 2 Q\right)}
$$

where we have lumped all dissipation and frequency shifts due to, for instance, coupling holes, beam tunnels, wall resistivity, etc., into the value of $Q$ and adjusted the resonant frequency $\omega_{n}$. The average energy stored in the cavity can be expressed in terms of $A$

$$
U=\frac{V}{4 \pi} \int_{0}^{\infty} \frac{d \omega d \omega^{\prime}}{(2 \pi)^{2}} A(\omega) A^{*}\left(\omega^{\prime}\right) e^{-i\left(\omega-\omega^{\prime}\right) t}+\text { c.c. }
$$

where we have assumed, according to (4), that $A(\omega)$ is peaked near $\omega_{n}$ and averaged over time corresponding to $2 \pi / \omega_{n}$ eliminating terms with approximate frequency $2 \omega_{n}$. The average power dissipated in the cavity is given in terms of the energy stored, the frequency, and the quality factor

$$
P=\frac{\omega_{n} U}{Q} \text {. }
$$

The next step of the calculation is to evaluate the numerator in (4). To do this we first write the time dependent current density in terms of the trajectory followed by individual increments of charge

$$
\boldsymbol{J}(\boldsymbol{x}, t)=\sum_{\delta Q} \delta Q \boldsymbol{v}_{\boldsymbol{o}}(t) \delta\left[x-x_{\circ}(t)\right]
$$

where $\delta Q$ represents an increment of charge which follows the trajectory $\left[\boldsymbol{x}_{o}(t), \boldsymbol{v}_{o}(t)\right]$. For the case of shot noise $\delta Q$ will represent the charge of an individual electron and the summation will include all electrons passing through the cavity. However, at this point we will allow flexibility in our treatment and leave the specification of $\delta Q$ for later. The trajectory will be calculated assuming that the high frequency fields in the cavity are weak and that the trajectory is unaffected by them. In this case, trajectories for particles with identical entrance momenta and coordinates are distinguished only by their entrance time. The current density can then be written

$$
\begin{gathered}
\boldsymbol{J}(\boldsymbol{x}, t)=\sum_{\delta Q} \frac{\delta Q\left(t_{e}\right)}{\boldsymbol{v}_{z}(z)} \boldsymbol{v}_{\boldsymbol{o}}(z) \delta\left[\boldsymbol{x}_{\perp}-\boldsymbol{x}_{\perp \circ}(z)\right] \\
\cdot \delta\left[\left(t-\tau(z)-t_{e}\right]\right.
\end{gathered}
$$

where we now express the orbit with $z$ as the independent variable and $\tau(z)$ represents the time to reach the point $z$ (measured from some reference surface, $z=0$ ). The variable $t_{e}$ is the time of entrance of the increment of charge $\delta Q$. This increment of charge can be related to the beam current entering at a particular time $I\left(t_{e}\right)$

$$
\sum_{\delta Q} \delta Q\left(t_{e}\right)=\int d t_{e} I\left(t_{e}\right)
$$

That is, we replace the sum over increments of charge by an integral over the time dependent beam current. Various temporal forms for this beam current can be assumed. For example, bare shot noise is calculated if one takes the beam current to consist of a train of delta functions with weight $e$, the electron charge, and random entrance times. Alternatively one could take the beam current to vary coherently in time. Both these and more will be considered subsequently.

We now take the Fourier transform with respect to time of (7), and insert it in the numerator of (4) to obtain for $A(\omega)$

$$
A(\omega)=-\frac{2 \pi i c \hat{I}(\omega) F(\omega)}{V\left(\omega-\omega_{n}+i \omega_{n} / 2 Q\right)}
$$

where $\hat{I}(\omega)$ is the Fourier transform of the time dependent beam current. The quantity $F(\omega)$ in (8)

$$
F(\omega)=\int d \tau \boldsymbol{E}_{n}^{*}\left[\boldsymbol{x}_{0}(\tau)\right] \cdot \boldsymbol{\beta}(\tau) e^{i \omega \tau}
$$

is an integral over time along the orbit. It represents the Fourier transform of the product of the relativistic factor, $\beta(\tau)=$ $\boldsymbol{v}_{o}(\tau) / c$, and the cavity electric field eigenfunction. A similar quantity appears in the expression for the power radiated per unit frequency and per unit solid angle into free space [6]. Generally, the quantity $F$ will have a peak value which scales as the product of the perpendicular component of $\beta$ times the time of flight through the cavity. Due to the oscillatory nature of $\beta_{\perp}$, this peak will occur for frequencies near the cyclotron frequency. Additionally, the value of $F$ will reflect how well the particles couple to the fields in the cavity. This time integral can be evaluated for a $\mathrm{TE}_{n p 1}$ mode in a circularly symmetric cavity of radius $r_{w}$ and length $L_{c}$. We take the axial profile of the electric field to be Gaussian with a full width at $1 / e$ of 
the maximum to be $L_{c}\left[\boldsymbol{E}_{n} \sim \exp \left(-4 z^{2} / L_{c}^{2}\right)\right]$. The resulting value of $F(\omega)$ is

$$
F(\omega)=\left(\frac{\pi}{2}\right)^{1 / 4} \frac{L_{c} \beta_{\perp} h}{2 v_{z}} \exp \left(-\delta^{2} / 4\right)
$$

where

$$
h=\frac{J_{n+1}\left(j_{n p}^{\prime} r_{b} / r_{w}\right)}{\left(1-n^{2} / j_{n p}^{\prime 2}\right)^{1 / 2} J_{n}\left(j_{n p}^{\prime}\right)}
$$

is the coupling coefficient, with $J_{n}$ the ordinary Bessel function, $r_{b}$ the annular beam radius, and $j_{n p}^{\prime}$ the zero of the derivative of the Bessel function. The frequency dependence of $F$ enters through the dimensionless detuning parameter $\delta$

$$
\delta=\left(\omega-\Omega_{c} / \gamma\right) \frac{L_{c}}{2 v_{z}}
$$

where $\Omega_{c} / \gamma$ is the relativistic cyclotron frequency. The quantity $F$ is, thus, peaked in frequency at the cyclotron frequency with a width in frequency determined by the particle transit time through the cavity.

We are now at a point where we can compare the power dissipated in the cavity for the case of a beam which is premodulated at a specified frequency and an unmodulated beam consisting of a stream of uncorrelated electrons. In the first case the time dependent beam current is given by $I(t)=I_{0}+\delta I \cos \omega_{0} t$. The Fourier transform for $\omega>0$ is thus, $\hat{I}(\omega)=\pi \delta I \delta\left(\omega-\omega_{0}\right)$. The resulting power dissipated in the cavity is given by

$$
P=\frac{\pi F^{2} c^{2}}{2 V} \frac{\omega_{n} / Q}{\left|\omega_{0}-\omega_{n}+i \omega_{n} / 2 Q\right|^{2}}(\delta I)^{2} .
$$

In circuit theory this formula corresponds to the power dissipated in the parallel combination of a resistor, capacitor, and inductor driven by an ideal current source $\delta I$. The equivalent shunt resistance is given by

$$
R\left(\omega_{0}\right)=\frac{4 \pi F^{2} c^{2}}{V \omega_{n} / Q}
$$

and the power dissipated at the resonance is $P=$ $(1 / 2) R\left(\omega_{n}\right)(\delta I)^{2}$. The values of the equivalent inductance and capacitance can then be determined by the relations $\omega_{n}=1 /(L C)^{1 / 2}$ and $Q=R(C / L)^{1 / 2}$.

The shunt resistance is frequency dependent through its dependence on $F$. In this sense, the simple circuit analogy doesn't quite apply. However, the concept of a shunt resistance, which is used to characterize cavities in accelerators and klystrons [7], is useful anyway as we shall see. In the electrostatic system of units (ESU), which we have used so far, resistance has the units of inverse velocity. To convert to ohms (SI) one multiplies by $c / 4 \pi$ and the impedance of free space, $\left(\mu_{0} / \varepsilon_{0}\right)^{1 / 2}=377 \Omega$

$$
R_{\Omega}=377 \frac{F^{2} c^{3}}{V \omega_{n} / Q}[\Omega] .
$$

For a $\mathrm{TE}_{n p 1}$ mode in a cylindrical cavity we can use expressions (10) and (14) to write the resistance

$$
R_{\Omega}=236 Q \frac{L_{c}}{\lambda}\left(1-\frac{\lambda^{2}}{4 L_{c}^{2}}\right) \frac{\alpha^{2} h^{2}}{j_{n p}^{\prime 2}} \exp \left(-\delta^{2} / 2\right)[\Omega]
$$

where $\alpha=v_{\perp} / v_{z}$ is the beam pitch factor and $\lambda$ is the wavelength based on the cavity resonance frequency. Thus, for typical parameters the shunt resistance is on the order of $100 Q \Omega$. For example, for a $\mathrm{TE}_{011}$ mode, with the beam placed on the maximum of the coupling coefficient, $\alpha=1, \delta=0$, and $L_{c} / \lambda=3$ we find $R_{\Omega}=97 Q \Omega$. An electron beam with a $2 \mathrm{~A}$ modulation injected into a cavity with a quality factor of 150 would then dissipate about $29 \mathrm{~kW}$ in the cavity. This number is comparable to the expected saturated power that would be extracted in the output cavity. This follows from the observation that in a good design the beam current is nearly fully modulated when it reaches the output cavity.

For the case of random electrons we need to compute the expected value of the product $I(\omega) I^{*}\left(\omega^{\prime}\right)$. This we do by first representing the current by a train of delta functions, $I(t)=e \sum_{i} \delta\left(t-t_{i}\right)$. This leads to the Fourier transform $\hat{I}(\omega)=e \sum_{i} \exp \left(i \omega t_{i}\right)$. We then form the expected value of the product of two Fourier transforms

$$
\left\langle\hat{I}(\omega) \hat{I}^{*}\left(\omega^{\prime}\right)\right\rangle=e^{2} \sum_{i, j}\left\langle\exp \left[i\left(\omega t_{i}-\omega^{\prime} t_{j}\right)\right]\right\rangle .
$$

We now assume that the entrance times are uncorrelated, and as a result, only terms with $i=j$ contribute to the sum. Then, assuming entrance times are uniformly distributed, we replace the sum and average with an integral over entrance time

$$
\begin{aligned}
\sum_{i}\left\langle\exp \left[i\left(\omega-\omega^{\prime}\right) t_{i}\right]\right\rangle & =\int_{-\infty}^{\infty} \frac{d t_{i}}{\Delta t} \exp \left[i\left(\omega-\omega^{\prime}\right) t_{i}\right] \\
& =\frac{2 \pi}{\Delta t} \delta\left(\omega-\omega^{\prime}\right)
\end{aligned}
$$

where $\Delta t=e / I_{0}$ is the mean time between electron arrivals, and $I_{0}$ is the average current (taken here to be positive). The result is the well known "white noise" power spectrum for a beam of uncorrelated electrons

$$
\left\langle\hat{I}(\omega) \hat{I}^{*}\left(\omega^{\prime}\right)\right\rangle=2 \pi e I_{0} \delta\left(\omega-\omega^{\prime}\right) .
$$

This expression, when inserted in (5) and (8), gives the following for the power dissipated in the cavity

$$
P=\frac{e I_{0}}{2} \int \frac{d \omega}{2 \pi} R(\omega) \frac{\left(\omega_{n} / Q\right)^{2}}{\left|\omega-\omega_{n}+i \omega_{n} / 2 Q\right|^{2}} .
$$

The shape of the spectral density of the power is thus determined by both the cavity resonance and the shunt resistance. We recall from (15) that the shunt resistance is peaked in frequency at the cyclotron frequency. The width of the shunt resistance resonance is $v_{z} / L_{c}$ while the width of the cavity resonance is $\omega_{n} / Q$. Thus, the detailed shape of the spectral density of the power will vary depending on the widths and central frequencies of the two resonance functions.

The total amount of noise power (integrated over frequency) will now be estimated in two limiting cases. In the first case we assume that the cyclotron resonance is much broader than the cavity resonance. This requires $Q L_{c} \omega_{n} / v_{z} \gg 1$. In this case, carrying out the integral in the above gives

$$
P=\frac{1}{2} R\left(\omega_{n}\right)\left(e \omega_{n} / Q\right) I_{0}
$$


From (17) it is clear that in this case the noise power is spread out over a range of frequencies corresponding to the $Q$ width of the input cavity.

The power given by (18) can be compared with the form that applies in the case of coherent excitation by noting, $e \omega_{n} / Q=I_{0} / N$, where $N$ is the number of electrons that pass through the cavity in a cavity decay time $Q / \omega_{n}$. A typical vale of $N$ is $10^{10}$. Thus, the noise power is about ten orders of magnitude smaller than the amount of power that could be driven by a coherently modulated beam. Since the later power corresponds roughly to the saturated output power in the device and since noise is amplified as the signal proceeds through the device, a very rough rule for the signal to noise ratio measured in $\mathrm{dB}$ is

$$
\text { Signal/Noise }=10 \log _{10} N-G[\mathrm{~dB}]
$$

where $G$ is the gain of the amplifier. Equation (19) is really only approximate as the parameters of the input and output cavities might be quite different. Also, it compares the signal power which may be in a very narrow band to the noise power which will be spread out over the resonance width of the cavity. More properly the signal to noise ratio is found by comparing (18) with drive power coupled into the first cavity.

In the second case of a narrow cyclotron resonance, $Q L_{c} \omega_{n} / v_{z} \ll 1$, the noise power can be estimated using the form (15) for the shunt resistance, one obtains

$$
P=\frac{1}{2}\left(\frac{\pi}{2}\right)^{1 / 2} R(0) \frac{\left(\omega_{n} / Q\right)^{2}}{\left|\Omega_{c} / \gamma-\omega_{n}+i \omega_{n} / 2 Q\right|^{2}}\left(e L_{c} / v_{z}\right) I_{0} .
$$

In this case the detuning between the cyclotron frequency and cavity frequency is introduced into the cavity resonance function and the noise power is spread out over a range of frequencies corresponding to the inverse of the particle transit time, $v_{z} / L_{c}$. Further, one can introduce a dimensionless number of electrons $N$ based on the transit time $e L_{c} / v_{z}=$ $I_{0} / N$. Generally, the smaller of (18) and (20) is applicable.

An alternate way of characterizing the noise is in terms of the noise temperature. According to the definition, the noise temperature is the power per unit frequency [in $\mathrm{Hz}$ ] dissipated in the cavity. From (17) this is seen to be

$$
T(\omega)=\frac{1}{2} R(\omega) e I_{0} \frac{\left(\omega_{n} / Q\right)^{2}}{\left|\omega_{0}-\omega_{n}+i \omega_{n} / 2 Q\right|^{2}} .
$$

At resonance the temperature $T\left(\omega_{n}\right)$ is given by

$$
T\left(\omega_{n}\right)=2 R\left(\omega_{n}\right) e I_{0}=4\left(e / I_{0}\right) \times \frac{1}{2} R\left(\omega_{n}\right) I_{0}^{2} .
$$

According to our previous discussions, the last factor on the right hand side, $1 / 2 R I_{0}^{2}$ (with $R$ replaced by the resistance of the output cavity), is essentially the maximum output power the beam could deliver. This is a good fraction of the beam power $V_{0} I_{0}$. The temperature, therefore, corresponds roughly to the energy of the electron beam. This may seem surprising at first since our beam is cold, but a spiraling electron beam is far from thermodynamic equilibrium and has an available free energy which for $\alpha \approx 1$ scales as the beam energy. This interpretation is enforced by noting that the resistance $R$ is proportional to $\alpha^{2}$, and thus vanishes if the free energy of the beam is removed.

For some radar applications the phase of the returning signal is detected. There will be a number of contributions to phase noise including the inherent noise of the transmitter. To characterize the effect of shot noise on the noise in the transmitted signal phase we express the time dependent amplitude of the signal in the bunching cavity

$$
A_{s}(t)=A_{0}+\int_{0}^{\infty} \frac{d \omega}{2 \pi} A(\omega) e^{i\left(\omega_{0}-\omega\right) t} .
$$

Here, $A_{s}$ is the complex amplitude of the total signal, $A_{0}$ is the complex amplitude of the coherent signal at frequency $\omega_{0}$, and $A(\omega)$ is the Fourier transform of the noise. The time dependent phase is then given by $\delta \Phi=\arg \left\{A_{s} A_{0}^{*}\right\}$. For low levels of noise this gives

$$
\delta \Phi(t)=\operatorname{Im}\left\{\int_{0}^{\infty} \frac{d \omega}{2 \pi} \frac{A(\omega) A_{0}^{*}}{\left|A_{0}\right|^{2}} \exp \left[-i\left(\omega-\omega_{0}\right) t\right]\right\} .
$$

The fluctuations, in phase can then be characterized by a correlation function

$$
\begin{aligned}
\langle\delta \Phi(t) \delta \Phi(t+\tau)\rangle=\frac{1}{2} \operatorname{Re}\{ & \int_{0}^{\infty} \frac{d \omega d \omega^{\prime}}{(2 \pi)^{2}}\left\langle\frac{A(\omega) A^{*}\left(\omega^{\prime}\right)}{\left|A_{0}\right|^{2}}\right\rangle \\
& \left.\cdot e^{\left[i\left(\omega_{0}-\omega\right) t+i\left(\omega-\omega_{0}\right)(t+\tau)\right]}\right\} .
\end{aligned}
$$

Using the expression (5) for the energy density in the cavity, along with the definition of the noise temperature (21), and the expression the coherent power coupled into the cavity $P_{0}=\omega_{n} V\left|A_{0}\right|^{2} /\left(2 \pi Q_{\omega}\right)$ where $Q_{\Omega}$ is the ohmic $Q$ value (not including the coupling hole) it is possible to express the phase noise correlation function

$$
\langle\delta \Phi(t) \delta \Phi(t+\tau)\rangle=\frac{Q}{2 Q_{\Omega}} \operatorname{Re}\left\{\int_{0}^{\infty} \frac{d \omega}{2 \pi} \frac{T(\omega)}{P_{0}} e^{\left[-i\left(\omega-\omega_{0}\right) \tau\right]}\right\} .
$$

Thus, the phase noise power spectrum, which is the Fourrier transform of the correlation function, is the ratio of the noise temperature to signal power coupled into the bunching cavity, times the ratio of the total $Q$ to the twice the ohmic $Q$.

\section{Collective EfFects}

Expression (17) for the power spectrum of the beam current was derived assuming that the electrons entering the cavity are uncorrelated with one another. This is an assumption which most likely is not valid. Experience with sources of microwaves driven by linear beams has shown that the electrons can become highly correlated and this reduces substantially the noise in the beam. An excellent review of the early studies of shot noise in devices with linear beams can be found in [2] and [8]. We will review here some important results, but presented from an alternate point of view. Within the framework of plasma kinetic theory the correlations occur because of the dielectric shielding of the discrete electron charges by the beam itself. We can consider this effect by analogy to the case of electrostatic plasma waves treated in text books [9]. By working in either the spatial or Fourier domain one can show that the shot noise is generated by shielded 
rather than bare electrons. The end result is that the power spectrum of the noise is modified by a term representing the dielectric shielding. For example, we modify expression (21) by noting that the shunt resistance depends on the velocity space coordinates of the electron beam. Thus, if the beam has a distribution of velocities we expect that the shunt resistance $R(\omega)$ should be redefined as

$$
R(\omega) \rightarrow \int d^{3} v \frac{v_{z}}{v_{0}} f(v) \frac{R(\omega, v)}{\left|1+\chi\left(\omega, k_{d}\right)\right|^{2}}
$$

where $f(v)$ is the velocity space distribution function for the beam, $v_{0}$ is the average axial velocity of the beam, $\chi(\omega, k)$ is the beam susceptibility (we assume a one-dimensional (1-D) model of the self fields), and $k_{d}(\boldsymbol{v})=\omega / v_{z}$, is the Doppler wave number for electrons of velocity $v$ interacting with fields of frequency $\omega$. The factor $v_{z} / v_{0}$ accounts for the fact the product $I_{0} R$ is what enters expression (17). Thus, if we were to consider the beam to be composed of many beamlets the distribution function should be weighted by the axial velocity.

The shielding effect occurs when the beam susceptibility $\chi$ is large. Generally, this requires a combination of sufficiently high beam density and sufficiently low beam temperature. To illustrate this we consider for the moment the case of a linear beam. We ignore the velocity dependence of the shunt resistance and calculate a reduction factor $H(\omega)$

$$
H(\omega)=\int d^{3} v \frac{v_{z}}{v_{0}} \frac{f(\boldsymbol{v})}{\left|1+\chi\left(\omega, k_{d}\right)\right|^{2}}
$$

where we have used $k_{d}=\omega / v_{z}$ for the Doppler wave number. Let us further take the beam distribution function to be Maxwellian with mean velocity $v_{0}$ and temperature $T_{b}=(1 / 2) m v_{t}^{2}$

$$
f\left(v_{z}\right)=\left(\frac{m}{2 \pi T_{b}}\right)^{1 / 2} \exp \left[-\frac{m\left(v_{z}-v_{0}\right)^{2}}{2 T_{b}}\right] .
$$

For this distribution the beam susceptibility can be expressed in terms of the plasma dispersion function $Z(\zeta)$

$$
\chi(\omega, k)=\frac{2 \omega_{p}^{2}}{k^{2} v_{t}^{2}}\left[1+\frac{\omega-k v_{0}}{|k| v_{t}} Z\left(\frac{\omega-k v_{0}}{|k| v_{t}}\right)\right] .
$$

We emphasize again that we are using a 1-D model of the self fields. Thus, the quantity $\omega_{p}$ represents the reduced plasma frequency which accounts for the transverse structure of the space charge fields.

The integral in (24) can be evaluated approximately in the limit of low beam temperature. That is, $k^{2} v_{t}^{2}=\omega^{2} v_{t}^{2} / v_{0}^{2} \ll$ $2 \omega_{p}^{2}$. There are two types of contribution to the integral. First, for velocities $v_{z}$ which are in the thermal distribution, $\left|v_{z}-v_{0}\right| \approx v_{t}$, the susceptibility is large, $\chi \gg 1$, and the contribution to the reduction factor scales as

$$
H \approx\left(\frac{\omega^{2} v_{t}^{2}}{2 \omega_{p}^{2} v_{0}^{2}}\right)^{2} \approx \frac{\omega^{4}}{\omega_{p}^{4}} \frac{T_{b}^{2}}{E_{b}^{2}} .
$$

Where $E_{b}=(1 / 2) m v_{0}^{2}$ is the beam energy. For a cold dense beam, this is a large reduction. A second contribution comes from velocities for which $1+\chi \cong 0$; in other words, from velocities which resonantly interact with beam space charge waves. For a relatively cold beam these velocities are in the tail of the distribution function so the numerator in the integral is very small. However, the imaginary part of the susceptibility is also very small and the contribution to the integral is nonnegligible. This contribution can be found by expanding the denominator around the two velocities corresponding to excitation of the slow and fast space charge waves. The result for the contribution to the reduction factor is

$$
H(\omega)=\frac{n \omega T_{b}}{2 \omega_{p} E_{b}}
$$

where $n=1$ or 2 depending on whether there are one or two forward propagating space charge modes where the denominator in (24) becomes very small. This contribution dominates that of the thermal particles if $T_{b} / E_{b}<\left(\omega_{p} / \omega\right)^{3}$. In this limit the noise temperature at resonance (22) is given by

$$
T\left(\omega_{n}\right)=n \frac{R\left(\omega_{n}\right)}{Z_{0}} T_{b}
$$

where $Z_{0}=2\left(\omega_{p} / \omega\right) E_{b} /\left(e I_{o}\right)$ is a characteristic impedance. In fact, one can show that $Z_{0}$ is the characteristic impedance of the space charge modes [8]. That is, a beam that supports a small space charge wave will have fluctuations in both voltage and current. The ratio of the voltage fluctuation to the current fluctuation is $Z_{0}$. In the case in which the cavity and space charge waves have comparable impedances the noise temperature is equal to the beam temperature. Further, the dominant current fluctuations are in the form of weakly damped space charge modes as opposed to ballistic perturbations associated directly with the motion of individual particles. In linear beam devices these fluctuations are imposed near the cathode where the beam is dense and the mean speed is comparable to the thermal speed. That is, the Landau damping of plasma waves is sufficiently weak once the beam has been accelerated that the waves and beam are not strongly coupled. That is the temperature $T_{b}$ is determined by the cathode temperature and the linear propagation of plasma waves on the accelerating beam as they travel from the cathode to the interaction region.

In the preceding discussion we evaluated the susceptibility for a 1-D, linear beam. The dominant collective effect in this case is the excitation of beam space charge modes: i.e., plasma waves. The beam in a magnetron injection gun (MIG) is spiraling about the magnetic field lines and a more appropriate susceptibility takes into account the possibility of cyclotron resonance. For example, the susceptibility of a warm beam, responding to transverse electrostatic fields with frequencies near the cyclotron resonance can be found in [10]

$$
\chi(\omega, k)=\frac{\beta_{\perp 0}^{2} \gamma_{0}^{3} \omega_{p}^{2}}{4 \gamma_{z 0}^{2} \Omega_{c}^{2}}\left(\omega^{2}-k^{2} c^{2}\right) \int \frac{d \gamma d u_{z} f\left(\gamma, u_{z}\right)}{\left(\gamma \omega-k u_{z}-\Omega_{c}\right)^{2}}
$$

where $\gamma_{0}=$ is the mean relativistic factor, $\gamma_{z 0}=[1-$ $\left.\left(v_{0} / c\right)^{2}\right]^{-1 / 2}$ where $v_{0}$ is the mean axial velocity of the beam and $\beta_{\perp 0}$ is the mean transverse relativistic factor for the beams perpendicular velocity. This expression assumes $k$ is the axial wave number, and that the perturbations also have a perpendicular wave number which is much greater than $k$. However, in this approximation, the value of the 
perpendicular wavenumber does not appear in the expression for the susceptibility (see [10] for details). The function $f\left(\gamma, u_{z}\right)$ describes the beam's distribution of energy and axial momentum. In principle, one should include the variations of perpendicular momentum under the integral. However, when the spread in the various momenta is small, as assumed here, only the variation of quantities which appear in the resonant denominator needs to be taken into account. Let us now assume that the beam is monoenergetic but with a distribution that depends on axial momentum. For ease of integration we take the distribution of axial momenta to be Loretzian with mean velocity $v_{0}$ and thermal velocity $v_{t}$

$$
f\left(\gamma, u_{z}\right)=\frac{v_{t} \delta\left(\gamma-\gamma_{0}\right)}{\pi \gamma_{0}\left[\left(u_{z} / \gamma_{0}-v_{0}\right)^{2}+v_{t}^{2}\right]} .
$$

In this case the susceptibility can be evaluated in terms of simple functions

$$
\chi(\omega, k)=\frac{\beta_{\perp 0}^{2} \gamma_{0} \omega_{p}^{2}}{4 \gamma_{z 0}^{2} \Omega_{c}^{2}} \frac{\omega^{2}-k^{2} c^{2}}{\left(\omega-\Omega_{c} / \gamma_{0}-k v_{0}+i k v_{t}\right)^{2}} .
$$

The sign of the term $\omega^{2}-k^{2} c^{2}$ in the numerator of the expression for $\chi$ is of significance. The factor $\omega^{2}$ can be attributed to gyrophase bunching of the beam while the term $k^{2} c^{2}$ is attributable to axial bunching [11]. If $\omega^{2}>k^{2} c^{2}$ gyrophase bunching dominates and electrostatic cyclotron modes can be unstable. The axial wavenumber of the cyclotron mode with frequency $\omega$ is determined by setting $1+\chi=0$. It can be written as the Doppler wavenumber based on the mean velocity of the beam plus a correction

$$
k=\frac{\omega-\Omega_{c} / \gamma_{0}}{v_{0}}+\delta k
$$

In the limit of a tenuous beam, $\beta_{\perp 0}^{2} \gamma_{0} \omega_{p}^{2} \ll 4 \gamma_{z 0}^{2} \Omega_{c}^{2}$, the small wave number shift $\delta k$ is given by

$$
\delta k= \pm i k_{I}+i k_{t}
$$

where

$$
k_{I}^{2}=\frac{\gamma_{0} \omega_{p}^{2} \alpha^{2}}{4 \gamma_{z 0^{2}}^{2} \Omega_{c}^{2}}\left[\frac{\omega^{2}}{c^{2}}-\frac{\left(\omega-\Omega_{c} / \gamma_{0}\right)^{2}}{v_{0}^{2}}\right]
$$

and

$$
k_{t}=\frac{\left|\omega-\Omega_{c} / \gamma_{0}\right| v_{t}}{v_{0}^{2}}
$$

The quantity $k_{I}$ represents the spatial growth rate of the electrostatic cyclotron instability for a cold beam, while $k_{t}$ represents a damping rate due to axial velocity spread. The importance of the electrostatic cyclotron instability was first recognized by Hirshfield [12] and Charbit et al. [13]. Subsequently, linear and nonlinear analyzes of the instability [14]-[16] have been carried out in which its effect on current fluctuations and energy spread were considered. Of particular concern was the possible presence of this instability in beam drift tubes. According to (35a) the spatial growth rate is maximum at cyclotron resonance where $k_{I}$ reaches its maximum value and $k_{t}$ vanishes. The maximum growth rate occurs at cyclotron resonance because the Doppler wave number vanishes. Consequently, axial bunching is negligible [10] and

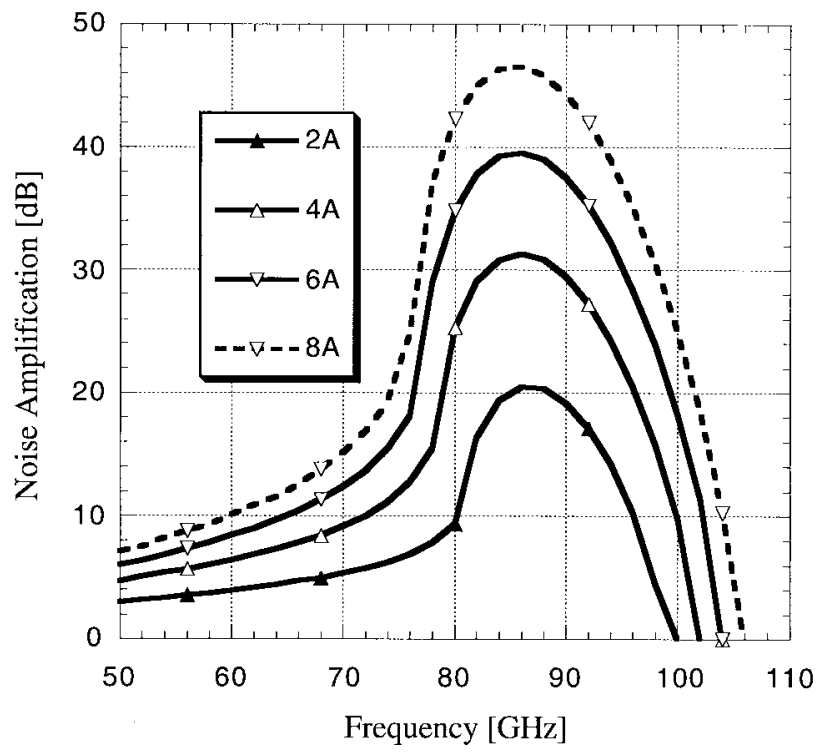

Fig. 1. Noise amplification [17] versus frequency for the parameters of the NRL $94 \mathrm{GHz}$ gyroklystron.

the resonant denominator in (30) is insensitive to the spread in axial velocities [15].

In a MIG the magnetic field typically varies by a factor of 10 to 30 in the drift region between the cathode and the cavity. This has a large effect on the amount of spatial amplification a fluctuation with fixed frequency will experience, as was recognized in [15] and quantified in [10]. Fluctuations with frequencies close to the cyclotron frequency in the input cavity, where the magnetic field is near its maximum, will be damped in the drift region up to a point where the magnetic field is close to its value in the cavity. At this point the fluctuations become unstable and grow exponentially. Thus the amount of noise will be largely affected by the amount of growth that occurs in these last few centimeters [10]. The growth length of the instability is also on the order of a few centimeters. Therefore, large enhancements in the noise level are possible. For example, Fig. 1 [17] shows the expected amount of noise power amplification due to the electrostatic cyclotron instability for the beam parameters of the NRL gyroklystron [1]. Plotted is the number of decibels of growth versus frequency for a several different beam currents. For these calculations the beam was assumed to have a "top hat" dependence on axial velocity as opposed to Lorenzian. The resulting expression for the wavenumber is given by (27) of [10]. As can be seen, for the highest currents nearly $50 \mathrm{~dB}$ of amplification is expected.

Formula (23) for the effect of dielectric shielding assumes that the plasma is stable, and can not be applied for cases in which the instability is present. A more refined theory is required to describe the growth of current fluctuations on the beam as it passes from a region where the beam is stable to one where the beam is unstable. Presumably fluctuations start at a level given by (23) and then grow exponentially after the beam becomes unstable. At the point where the beam becomes unstable the susceptibility is of order unity, since 1 $+\chi=0$. Thus the noise may grow from a level for which 
the dielectric shielding is unimportant. This was the situation assumed in [10]. However, a more precise prediction awaits the development of a theory which treats the evolution of noise fluctuations on an inhomogeneous beam.

An additional collective effect which is easily incorporated in the present theory is the coherent response of the electron beam to the fields in the cavity. In particular, we have calculated the fields in the cavity assuming the current perturbation is prescribed. These fields will produce a coherent response in the beam which is nonnegligible if the beam is close to the value required to start oscillations in the cavity. The result is that all the resonant denominators appearing in (4), (8), (12), (17), (20), and (21) need to be modified to include the complex frequency shift induced by the beam. That is the following replacement should be made [5]

$$
\omega-\omega_{n}+i \omega_{n} / 2 Q \rightarrow \omega_{0}-\omega_{n}+i \omega_{n} / 2 Q-\Delta \omega
$$

where after a small calculation the frequency shift is written

$$
\Delta \omega=-\frac{2 \pi i \operatorname{Ie}}{\mathrm{mV}} \int d^{3} v f \frac{\partial}{\beta_{\perp} \partial \beta_{\perp}}\left(\int_{-\infty}^{\infty} d \tau F_{\tau} \frac{d}{d \tau} F_{\tau}^{*}\right)
$$

The integral over $d^{3} v f$ in (37) is an average over initial particle momenta, while the integral over $\tau$ is carried out along the unperturbed particle orbit. The quantity $F_{\tau}$ is defined

$$
F_{\tau}\left(\omega, \beta_{\perp}\right)=\int_{-\infty}^{\tau} d \tau^{\prime} \boldsymbol{E}_{n}^{*}\left[\boldsymbol{x}_{0}\left(\tau^{\prime}\right)\right] \cdot \beta\left(\tau^{\prime}\right) e^{i \omega \tau^{\prime}} .
$$

Thus, according to (9) $F_{\infty}=F(\omega)$. Using (38) the imaginary part of the frequency shift can be expressed in terms of the shunt resistance

$$
\operatorname{Im}\{\Delta \omega\}=-\frac{\omega_{n}}{2 Q} \frac{I e}{m c^{2}} \int d^{3} v f \frac{\partial R\left(\omega, \beta_{\perp}\right)}{2 \beta_{\perp} \partial \beta_{\perp}} .
$$

Expression (39) reiterates the known relation between the linear gain and the spontaneous emission rate. In the case of free electron lasers this is known as Madey's theorem. It has been derived previously for gyrotrons by Latham [18].

\section{CONCLUSION}

The excitation of noise in the input of a gyroklystron cavity can be expressed in terms of the excitation of a resonant circuit, with an equivalent frequency dependent shunt resistance, by the random fluctuations of the beam current. A typical value of the shunt resistance is $100 Q \Omega$ where $Q$ is the quality factor of the cavity. For the case of spontaneous emission, which neglects correlations in the entrance times of particles, the noise spectrum has two peaks, one at the cyclotron resonance and one at the cavity resonance. The noise temperature in the case of spontaneous emission scales as the beam energy.
Collective effects tend to reduce the noise level in linear beams to a value dependent on the beam temperature. However, in the case of a spiraling beam such as that produced by a MIG the noise can be enhanced by many $\mathrm{dB}$ due to the presence of unstable electrostatic cyclotron modes on the beam.

\section{REFERENCES}

[1] M. Blank, B. G. Danly, B. Levush, P. E. Latham, and D. Pershing, "Experimental demonstration of a W-band gyroklystron amplifier," Phys. Rev. Lett., vol. 79, p. 4485, 1997.

[2] L. D. Smullin and H. A. Haus, Eds., Noise in Electron Devices. New York: Wiley, 1959.

[3] G. S. Nusinovich and O. Dumbrajs, "Technical noise in gyroklystrons and phase-locked gyrotron oscillators," Phys. Plasmas, vol. 4, pp. 1424-1433, 1997.

[4] R. E. Collin, Foundation for Microwave Engineering, 2nd ed. New York: McGraw-Hill, 1992, ch. 7.

[5] M. Garven, W. Manheimer, and M. Blank, "Simple theory of input couplers for gyroklystron amplifiers," this issue, pp. 433-443.

[6] J. D. Jackson, Classical Electromagnetic Theory, 2nd ed. New York: Wiley, 1975, chs. 8 and 9.

[7] G. A. Loew and R. Talman, "Lectures on the elementary principles of linear accelerators," in Physics of High Energy Particle Accelerators, AIP Conf. Proc. No. 105, M. Month, Ed. New York: American Institute of Physics, 1983, p. 15.

[8] J. R. Whinnery, "History and problems of microwave tube noise," Scienta Electra, vol. 5, pp. 133-150, 1959.

[9] N. A. Krall and A. W. Trivelpiece, Principles of Plasma Physics. San Francisco, CA: San Francisco Press, 1986, pp. 556-567.

[10] H. Li and T. M. Antonsen, Jr., "Space charge instabilities in gyrotron beams," Phys. Plasmas, vol. 1, pp. 714-729, 1994.

[11] Expression (30) is derived from the plasma dielectric function in [10]. From this derivation one can trace the origin of the two terms contributing to the dielectric. The term proportional to $k^{2}$ comes from the axial electric field and the momentum dependence of the Doppler shift, while the term proportional to $\omega^{2}$ comes from the transverse electric field and the energy dependence of the cyclotron frequency. Thus, these terms can be attributed to axial and gyrophase bunching, respectively.

[12] J. L. Hirshfield, "Cyclotron harmonic maser," Int. J. Inf. Millim. Waves, vol. 2, p. 695, 1981 .

[13] P. Charbit, A Herscovici, and G. Mourier, "A partly self- consistent theory of the gyrotron," Int. J. Electron., vol. 51, p. 303, 1981.

[14] K. R. Chen and K. R. Chu, "Study of a noise amplification mechanism in gyrotrons," IEEE Trans. Microwave Theory Tech., vol. MTT-34, p. $72,1986$.

[15] A. Bondeson and T. M. Antonsen, Jr., "Space charge instabilities in gyrotron beams," Int. J. Electron., vol. 61, p. 855, 1986.

[16] K. R. Chu and L. H. Lyu, "Simulation of electrostatic noise amplification in gyrotrons," IEEE Trans. Microwave Theory Tech., vol. MTT-34, p. 690, 1986.

[17] S. Cooke, private communication.

[18] P. Latham, private communication.

Thomas M. Antonsen, Jr., for a biography, see this issue, p. 425.

Wallace M. Manheimer (M'85-SM'86-F'93), for a biography, see this issue, p. 443 . 